\title{
P-glycoprotein-mediated resistance to chemotherapy in cancer cells: using recombinant cytosolic domains to establish structure- function relationships
}
A. Di Pietro, G. Dayan,
G. Conseil, E. Steinfels,
T. Krell, D. Trompier,
H. Baubichon-Cortay and J.-M. Jault

\author{
Laboratoire de Biochimie Structurale et Fonctionnelle, \\ Institut de Biologie et Chimie des Protéines, UPR 412-CNRS, Lyon, France
}

\author{
Correspondence \\ A. Di Pietro \\ Laboratoire de Biochimie \\ Structurale et Fonctionnelle \\ Institut de Biologie et \\ Chimie des Protéines, UPR 412-CNRS \\ 7, Passage du Vercors \\ 69367 Lyon Cedex 07 \\ France \\ Fax: + 33-478-61-7873 \\ E-mail: a.dipietro@ibcp.fr \\ Research supported by CNRS \\ (UPR 412 and PCV97-129), Université \\ Claude Bernard-Lyon I, Fondation \\ de France (N o. 96003891), Région \\ Rhône-Alpes (Emergence 97027309), \\ Association pour la Recherche contre \\ le Cancer (No. 9147), Ligue \\ Nationale Contre le Cancer, \\ Fondation pour la Recherche \\ Médicale (No. 20000400-01)
}

Received March 18, 1999

Accepted May 7, 1999

\section{Abstract}

Resistance to chemotherapy in cancer cells is mainly mediated by overexpression of P-glycoprotein (Pgp), a plasma membrane ATPbinding cassette $(\mathrm{ABC})$ transporter which extrudes cytotoxic drugs at the expense of ATP hydrolysis. Pgp consists of two homologous halves each containing a transmembrane domain and a cytosolic nucleotide-binding domain (NBD) which contains two consensus Walker motifs, A and B, involved in ATP binding and hydrolysis. The protein also contains an $\mathrm{S}$ signature characteristic of $\mathrm{ABC}$ transporters. The molecular mechanism of Pgp-mediated drug transport is not known. Since the transporter has an extraordinarily broad substrate specificity, its cellular function has been described as a "hydrophobic vacuum cleaner". The limited knowledge about the mechanism of Pgp, partly due to the lack of a high-resolution structure, is well reflected in the failure to efficiently inhibit its activity in cancer cells and thus to reverse multidrug resistance (MDR). In contrast to the difficulties encountered when studying the full-length Pgp, the recombinant NBDs can be obtained in large amounts as soluble proteins. The biochemical and biophysical characterization of recombinant NBDs is shown here to provide a suitable alternative route to establish structure-function relationships. NBDs were shown to bind ATP and analogues as well as potent modulators of MDR, such as hydrophobic steroids, at a region close to the ATP site. Interestingly, flavonoids also bind to NBDs with high affinity. Their binding site partly overlaps both the ATP-binding site and the steroid-interacting region. Therefore flavonoids constitute a new promising class of bifunctional modulators of Pgp.
Key words

- P-glycoprotein (Pgp)

- Multidrug resistance

- Anticancer chemotherapy

- ATP

- Steroids

- Flavonoids 


\section{Anticancer chemotherapy: the problem of multidrug resistance}

Cancer cell resistance to cytotoxic drugs is considered to be one of the major obstacles to successful chemotherapy. Some tumors are intrinsically resistant to the treatments whereas others progressively acquire multidrug resistance (MDR) to a variety of structurally and functionally unrelated drugs including anthracyclines, Vinca alkaloids, epipodophyllotoxins, taxanes and actinomycin D.

The best studied mechanism of MDR (1) is the overexpression of P-glycoprotein (Pgp) (2), a 170-kDa plasma membrane protein that functions as an ATP-driven drug efflux pump in cancer cells. A family of Pgp isoforms is encoded by the $m d r$ genes which are located on human chromosome 7 , mouse chromosome 5 or Chinese hamster chromosome 1. Only the MDR1 isoform can confer the MDR phenotype to humans, whereas the two isoforms mdr1 (or mdr1b) and mdr3 (or mdr1a) in mice or pgp1 and pgp2 in Chinese hamsters can cause the same phenotype. An additional isoform, MDR2 (or MDR3) in humans, mdr2 in mice or pgp3 in Chinese hamsters, cannot confer MDR and appears to be involved in the transport of phospholipids, especially phosphatidylcholine $(3,4)$.

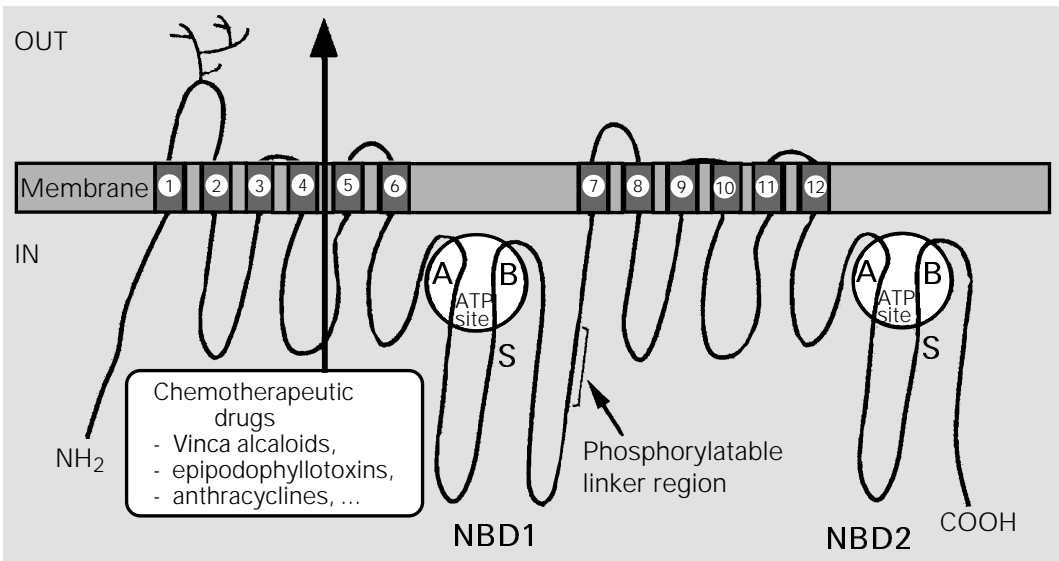

Figure 1 - Schematic structural organization of P-glycoprotein.

\section{The structure of Pgp: still a model}

cDNA sequences of human $M D R 1$, mouse $m d r 1$ and Chinese hamster $p g p 1$ have led to the prediction of protein secondary-structure elements and transmembrane segments which have allowed a topology model (Figure 1) to be established. The protein is composed of 1276 amino acids in mice, has a $141-\mathrm{kDa}$ molecular mass in the absence of glycosylation, and is composed of two homologous halves. The two halves share $43 \%$ sequence identity and $78 \%$ similarity and are most likely to have evolved as a result of gene duplication (5). Hydrophobicity analysis indicates that each half contains a hydrophobic domain with 6 transmembrane $\alpha$-helices (6) involved in chemotherapeutic drug efflux, and a cytosolic domain, nucleotide-binding domain 1 (NBD1) or NBD2, containing an ATP-binding site with characteristic Walker motifs A and B (7) and the S signature of $\mathrm{ABC}$ (ATP-binding cassette) transporters (8). A central sequence which connects the two homologous halves of the protein, called "linker region", contains in vitro phosphorylatable serine residues, and the first extracellular loop is heavily $N$-glycosylated.

A low-resolution structure $(2.5 \mathrm{~nm})$ has been obtained for Pgp by electron microscopy and single-particle image analysis (9). It shows a wide hydrophilic pore surrounded by a ring of transmembrane segments with a six-fold symmetry. The pore is closed on the cytosolic side by the two NBDs and the internal loops which connect the transmembrane spans. The cup-shaped chamber within the membrane has been proposed to include an opening allowing a lateral entry of drug substrates to be effluxed.

The crystal structure of HisP, the ATPbinding subunit of bacterial histidine permease, has recently been solved (10), and the structures of HisP and the NBDs are likely to be similar. HisP has an "L-shape" consisting of two thick arms: one side of arm 
I, including $\beta 1, \beta 2$ and $\beta 4-\beta 7$ strands (cf. Figure 4), is involved in dimerization, whereas the other side contains the ATPbinding pocket. The side-chain of a tyrosine residue located between $\beta 1$ and $\beta 2$ appears to form a stacking interaction with the adenine ring. The residues of the P-loop, containing the Walker motif $\mathrm{A}$, and of the following $N$-terminal segment of the $\alpha 1$ helix are mainly involved in the formation of hydrogen bonds with the ATP phosphates. Arm II contains helices $\alpha 3-\alpha 9$ which include the $\mathrm{S}$ signature, and is likely to interact with the membrane components of the transporter. The two arms of the $L$ are connected by a sixstranded $\beta$-sheet ( $\beta 3$ and $\beta 8-\beta 12$ ).

\section{ATPase activity and drug transport}

Although the exact mechanism of drug extrusion is not known, it is well established that Pgp functions as an ATP-dependent pump since: i) Pgp overexpression leads to both a lowered intracellular accumulation and an increased efflux of drugs and these effects are abolished by either intracellular ATP depletion or mutations within the Walker motifs A and B, ii) photoreactive drug analogues label Pgp, and iii) singlepoint mutations within either transmembrane spans or cytosolic loops differentially change the relative specificity towards transported drugs.

The ATPase activity of Pgp has been determined in enriched membranes of resistant cells and from a purified transporter reconstituted into liposomes. Both measurements resulted in values of a few micromoles/min $\mathrm{x} \mathrm{mg}$ protein for maximal rate and around $1 \mathrm{mM}$ for $\mathrm{K}_{\mathrm{m}}(\mathrm{MgATP})$. The maximal rate was dependent on the addition of phospholipids: it was either increased by phosphatidylethanolamine or phosphatidylcholine, or inhibited by phosphatidylserine or phosphatidylinositol. Pgp was found to be naturally associated with phosphatidylethanolamine and phosphatidylserine, but only with low amounts of phosphatidylcholine. Whether purified or inserted into plasma membranes, Pgps of different origins exhibit very similar maximal rates and catalytic properties. Both basal activity and stimulation by drugs or verapamil are dependent on the type of preparation and on its phospholipidic content. Some mutations and chemical modifications of transmembrane spans change both basal and drug-stimulated activities, indicating that the membrane components interact with the catalytic sites. Each of the two halves of Pgp exhibits a basal activity but their co-expression is required for drug-stimulated activity; therefore interactions between the two halves of the transporter are critical for its function (11). Since mutations in the Walker motif A of either NBD1 or NBD2 are sufficient to abolish drug efflux, interactions between the two NBDs are required for normal transporter activity (12). The hydrolysis of ATP probably leads to a high-energy conformation of the protein, the relaxation of which is coupled to drug transport. The two nucleotide-binding sites are able to hydrolyze ATP and appear to be strictly cooperative and alternative for catalytic activity (13).

Cancer cells with an increased expression of Pgp exhibit cross-resistance to a series of structurally unrelated drugs $(1,2,14)$. The only common features among these compounds are their hydrophobicity, their high size and a positive charge at neutral $\mathrm{pH}$ generally carried by a nitrogen atom, as determined by comparing colchicine derivatives (15). Two positively cooperative sites, with different but overlapping specificities, are likely to be involved in drug binding and efflux (16). There is evidence by affinity labelling that one of them is partly located on the cytosolic side of the transporter. The extrusion of the drugs from the inner leaflet of the plasma membrane strongly suggests a "hydrophobic vacuum cleaner" mechanism (17) for both sites. A flippase mechanism, involving substrate translocation outside the pore from the inner to the outer leaflet, has 
also been proposed (18), but only demonstrated for the mdr2/MDR2 transporter which uses phosphatidylcholine as a substrate $(3,4)$. The stoichiometry between transported substrate and hydrolyzed ATP was generally found to be close to unity (19) for a broad range of substrates such as colchicine, ${ }^{86} \mathrm{Rb}^{+}-$ valinomycin, vinblastine, daunorubicin or rhodamine 123.

\section{The physiological role of Pgp}

The pattern of distribution of the isoforms in different tissues has provided information about their physiological role. High levels of gene and protein expression of human MDR1 were detected in medulla, adrenal glands, kidney, jejunum, colon, as well as in capillary endothelium from testes and blood-brain barrier. In contrast, the MDR2 isoform is almost exclusively located in the liver. In mice, mdr1 is mainly expressed in adrenal glands, placenta and gravid uterus, whereas mdr 3 is found in lung, digestive tube and blood-brain barrier, and mdr2 is restricted to liver and muscles.

Role in detoxification. Pgp is predominantly localized in apical membranes of epithelia, on the luminal surface of jejunum and colon, kidney proximal tubules, bile and pancreas canalicules, and secretory epithelial cells of the gravid endometrium. Such a polarized expression is consistent with a role in natural cellular detoxification. Transgenic mice with deletion of the $m d r 3$ gene show that the absence of Pgp from the blood-brain barrier endothelium is correlated to a high increase in brain intoxication by ivermectin, which did not cause any effect in control animals (20). In addition, intravenous injection of vinblastine leads to a 22 -fold increased accumulation in the brain due to a lowered efflux rate. Therefore, it is reasonable to conclude that a physiological function of Pgp is the extrusion of cell poisons in order to detoxify tissue. This function might be especially important in the capillary en- dothelium of the blood-brain barrier.

Transport of hormones. The location of mdr1 Pgp in pancreatic ductules, pregnant mouse endometrium and adrenal cortex suggests its involvement in the transport of normal substrates such as steroid hormones. Pgp is indeed able to transport cortisol, aldosterone, dexamethasone and corticosterone $(21,22)$.

Phospholipid translocation. Transgenic mice with a deleted $m d r 2$ gene exhibit severe hepatic disease due to progressive destruction of ductule and bile canalicular cells. A complete absence of phospholipids is observed in the bile of homozygous $m d r 2-/-$ mice. It has been proposed that the human MDR2 isoform might facilitate phosphatidylcholine translocation from the inner to the outer leaflet of canalicular plasma membranes. The phosphatidylcholine accumulated on the outer leaflet can then be complexed by biliary salts excreted by canalicular cells. A similar function has been proposed for mouse mdr2 (3).

\section{Post-translational modifications}

Glycosylation. The molecular mass of mature Pgp varies between 130 and 180 $\mathrm{kDa}$, depending on the species and the type of cells. Pgp is synthesized as a nonglycosylated precursor, with an apparent molecular mass of 120 to $140 \mathrm{kDa}$, which matures with a half-time of either 1-2 $\mathrm{h}$ in human cells or 20-30 min in mice. In human Pgp, all oligosaccharidic chains are $N$-glycosylated, but the nature and sequence of sugars are unknown (23). The sequences of Pgps from different species have allowed the prediction of 2 to 4 potential glycosylation sites; the exact number and position of sites differ between man and rodents but are always located in the first extracellular loop (cf. Figure 1). Nevertheless, drug transport and ATPase activity are not dependent on glycosylation (24). A series of mutants with altered glycosylation sites also show the same 
cross-resistance to drugs. However, the much lower occurrence of resistant cell lines suggests that glycosylation might contribute to accurate folding and targeting of Pgp to the plasma membrane. In contrast, glycosylation does not protect against proteolytic cleavage since the usual 18-h half-life of the transporter is not affected.

Phosphorylation. Among the first scientific findings about Pgp was the fact that it is present as a phosphorylated protein; therefore the extent of phosphorylation was then used as a marker for protein maturation. Since human MDR1 Pgp, either purified from resistant KB-V1 cells or overexpressed as a recombinant protein, was phosphorylated in vitro by protein kinase $\mathrm{C}$, the latter was proposed to modulate multidrug resistance in animal cells (25). Protein kinase A and other, less-characterized, protein kinases were also able to phosphorylate Pgp, whereas phosphatases 1 and $2 \mathrm{~A}$ are involved in dephosphorylation. Pgp phosphorylation, which was either increased by the addition of protein kinase activators or decreased by inhibitors, was assumed to be correlated to cell multidrug resistance by changing the rate of drug transport, modifying specificity towards transported drugs, or affecting the stability of the transporter. However, most of these effectors have a low specificity, and have been used at concentrations which are vastly superior to those needed to achieve a selective inhibition of protein kinases (26). In addition, recent experiments with mutated phosphorylatable serines have shown that phosphorylation/dephosphorylation processes do not play an essential role in Pgpmediated multidrug resistance (27), although it was suggested that the binding affinity of some drugs might depend on the state of phosphorylation. Despite the existence of about forty consensus sites within Pgp (25), the four serine residues that can be phosphorylated by protein kinases $\mathrm{A}$ and $\mathrm{C}$ are all clustered in the "linker region" present in the central part of the transporter sequence (cf.
Figure 1). This indicates that the linker region is quite accessible to protein kinases.

\section{Multidrug transporters belong to the family of $A B C$ transporters}

The ABC ("ATP-binding cassette") transporter superfamily is characterized by the simultaneous presence of the Walker motifs $\mathrm{A}$ and B, involved in ATP binding and hydrolysis, and of the $\mathrm{S}$ signature involved in transport. $\mathrm{ABC}$ transporters play an important role both in eukaryotes and prokaryotes, especially in pathologies such as parasitic diseases, mycoses, familial cholestasis, eye diseases, hyperinsulinemic glycemia, adrenoleukodystrophy, cystic fibrosis and cancer cell resistance to chemotherapeutic drugs, as well as bacterial resistance to antibiotics. More than one hundred $\mathrm{ABC}$ transporters, also called "traffic ATPases" (28), have been identified in different species such as bacteria, yeasts, protozoan parasites, insects, plants and higher animals (29). Transported substrates are very different, ranging from a large polypeptide (HlyB) to a simple ion ("cystic fibrosis transmembrane conductance regulator", CFTR) and include sugars (MalEFGK, AraFGH), amino acids (HisJMPQ, GlnHPQ) and cytotoxic drugs (multidrug transporters).

Structural and functional homology of $A B C$ transporters. All ABC transporters exhibit a common structural organization with a total of four domains, two of them cytoplasmic and two membranous, which are synthesized either separately as two, three or four individual subunits, mainly in prokaryotes, or fused to form a single large polypeptide, mainly in eukaryotes. The two hydrophobic domains are generally formed by six transmembrane spans (6), but exceptions include the Escherichia coli maltose permease where MalF contains eight spans and the Salmonella typhimurium histidine permease where both HisQ and HisM have five. The "multidrug resistance-associated protein" 
(MRP) transporter contains a third transmembrane domain with five transmembrane spans near the $N$-terminal end which is extracellular (30). The two cytosolic domains of about two hundred amino acids, NBD1 and NBD2, contain the ATP-binding sites with three consensus sequences conserved among species. The Walker motifs A and B (7) are characteristic of many ATP-binding proteins. Motif A is also called "glycine-rich loop" or "P-loop" and has the consensus sequence $\mathrm{GX}_{4} \mathrm{GKT} / \mathrm{S}$ (with $\mathrm{X}$ being any amino acid), which is involved in binding the ATP phosphates. Motif B, with the sequence $Z_{4} D$ (with $Z$ being any hydrophobic amino acid), forms a $\beta$-strand and the aspartate residue generally interacts with the magnesium ion chelated to the nucleotide phosphates. A third consensus motif, the "S signature" of $\mathrm{ABC}$ transporters (8), is found just upstream of motif $B$. This segment is about fifteen amino acids in length and generally starts with the sequence LSGG. There are other sequence similarities particularly when the $N$-terminal and $C$-terminal halves of the same transporter are compared. Like MRP, some ABC transporters have an additional domain: CFTR contains a regulatory $\mathrm{R}$ domain which changes conformation when phosphorylated, whereas MalK includes a $C$-terminal extension of which the function is unknown. The bacterial permeases contain a fifth, periplasmic, subunit which binds the substrate to be imported with high affinity and specificity.

$\mathrm{ABC}$ transporters are able to transport a substrate against its concentration gradient by using the energy generated by ATP hydrolysis. They are therefore expected to obey a common overall mechanism (31) despite the fact they are operating in either substrate import (bacterial permeases) or substrate export (multidrug transporters) or ion channelling (CFTR).

ABC multidrug transporters. In addition to Pgp, mammalian cancer cells use MRP1, a 190-kDa glycoprotein, to extrude cytotoxic drugs (30). MRP1 appears to have an even lower substrate specificity than Pgp and is also able to transport anionic compounds complexed to glutathione, glucuronate or sulfate. The role of several recently identified isoforms is still to be elucidated (32). The smaller-sized ARA transporter might be involved in resistance to anthracyclines.

In yeasts, multidrug resistance was first described in Saccharomyces cerevisiae as "pleiotropic drug resistance" (PDR) (33). Two families of multidrug transporters were genetically and biochemically characterized in S. cerevisiae (34). One family, exemplified by PDR5 and SNQ2, includes proteins which resemble Pgp except that the transmembrane domains follow, instead of preceding, the NBDs in the polypeptide sequence. The second family, including YCF1 and YOR1, appears to be more closely related to MRP and CFTR. A PDR5 homologue has been described for the pathogen Candida albicans.

In protozoan parasites, the resistance to chloroquine of Plasmodium falciparum responsible for malaria is due to a rapid efflux of the drug and involves several genes (35). The $p f m d r 1$ gene encodes the $162-\mathrm{kDa}$ "Pglycoprotein homologue 1" (Pgh1) transporter (36). This protein is only indirectly involved in the transport mechanism since it is thought to regulate the intralysosomal $\mathrm{pH}$ by means of chloride ion channelling (35). In contrast, a direct mechanism for the extrusion of quinine, mefloquine and halofantine has been demonstrated for the Pgh1 transporter. Two types of genes were found in Leishmania strains: ltpgp A in L. tarantolae and lmpgp A in L. major. Both genes are located in a small extrachromosomal circular DNA, called H circle, frequently amplified upon selection with a hydrophilic drug (37). The ltpgp A protein of L. tarantolae and tcpgp2 from Trypanosoma cruzi (38) show a significant sequence homology with MRP (39). The transporters encoded by lemdr 1 in L. enriettii, ldmdr 1 in L. donovani 
and ltmdr1 in L. tropica exhibit high sequence identity with mammalian Pgp (40).

In bacteria, the resistance of Lactococcus lactis to amphipathic organic cations is mediated by Lmr A, a "half ABC transporter" consisting of one transmembrane domain and one cytosolic domain. This protein functions as a homodimer and confers the same MDR phenotype as Pgp to transfected human cells (41). A similar transporter is found in a number of bacteria and might be involved in resistance to antibiotics.

\section{Failure to inhibit Pgp with modulators which are also substrates}

Many efforts have been undertaken in the search for compounds which are able to inhibit the Pgp-mediated efflux of drugs in order to restore their intracellular cytotoxicity in tumors. Those compounds are called reversers, modulators or chemosensitizers. A number of compounds are able to reverse multidrug resistance in vitro, such as calcium channel blockers, calmodulin antagonists, hydrophobic peptides, lysosomotrophic agents, protein kinase inhibitors, antibiotics, hormones, and flavonoids $(1,14,42)$. Numerous other compounds with unrelated structures are also able to modulate multidrug resistance such as ionophores, oligodeoxy (ribo)nucleotides, noncytotoxic analogues of anticancer agents like $\mathrm{N}$-acetyl-daunorubicin, vinblastine derivatives, or inhibitors of hypertension like reserpine. A comparison of structurally different modulators resulted in mainly three similarities required for action: a nitrogen heteroatom and two planar, aromatic, rings (43). The modulatory effects of reserpine analogues on Pgp was highly dependent on the relative orientation of the three components.

However, the mechanism of action of modulators is poorly known. Some of them are thought to interact with the drug-binding sites and thus behave as competitive inhibitors. Unfortunately, most modulators includ- ing verapamil, cyclosporin A, FK506 and others (42) are transported by Pgp. Therefore, they are not real inhibitors but rather behave as pseudo-substrates that only lower the rate of anticancer-drug efflux. Hence, a permanent effect would require high concentrations of such compounds which would cause unendurable side-effects, such as cardiotoxicity and hypotension for verapamil, or immunosuppression for cyclosporin A. Recent reviews have tried to classify all compounds known to be modulators (44). Steroids are an interesting series of compounds with differential effects: some, mainly hydrophilic compounds, behave as substrates and are transported by Pgp, whereas others, more hydrophobic derivatives, are not transported and modulate vinblastine efflux (45).

\section{Non-transported potential modula- tors: hydrophobic hormones and flavonoids}

As shown above, the main cause for the inefficiency of many modulators is their behavior as pseudo-substrates. This is the case for hydrophilic steroids such as cortisol, aldosterone, dexamethasone, corticosterone or triamcinolone $(21,22)$. The search for modulators which are not transported has therefore become an attractive target. Hydrophobic derivatives such as progesterone, megestrol acetate or medroxyprogesterone acetate (45) or the RU486 antiprogestin (46) were shown not to be transported but to behave as strong modulators of drug efflux. The modulatory effect of steroids is directly correlated to the hydrophobicity of their substituents. This finding is consistent with previous studies about phenothiazines. Hydrophobic antiestrogens such as tamoxifen or derivatives also bind with high affinity to Pgp and are not transported (47). The oral administration of tamoxifen to test animals resulted in a serum concentration of 3-10 $\mu \mathrm{M}$. This concentration was shown to produce substantial inhibitory effects in vitro. Co-ad- 
ministration with adriamycin, vinblastine, etoposide or doxorubicin indeed produced a positive cell chemosensitization to the drug.

Flavonoids constitute another class of modulators thought not to be transported by Pgp. They are natural compounds present in plants which are known to exhibit general antiproliferative effects against cancer cells with low in vivo intrinsic cytotoxicity (48). Flavonoids have been reported to differentially affect cytotoxic drug efflux from cancer cells: hydroxylated compounds such as galangin, kaempferol and quercetin were found to increase the efflux of 7,12dimethylbenz $(a)$ anthracene or adriamycin (49), whereas quercetin and hydrophobic derivatives inhibited the efflux of rhodamin 123 (50) and Hoechst 33342 (51). Flavonoid inhibitors were found to bind to the ATP sites of several other ATP-utilizing proteins. This is certainly due to the structural similar- ity between flavonoids and the adenine moiety of ATP as demonstrated by crystallographic studies $(52,53)$. Those findings strongly suggest that the ATP sites of Pgp also serve as binding sites for flavonoids, and therefore it can be assumed that flavonoids are not transported by Pgp. In experiments using purified reconstituted Pgp, the flavonoid quercetin was shown both to inhibit ATPase activity (51) and to bind to two cooperative drug-binding sites; this resulted in either inhibition of Hoechst 33342 transport or stimulation of rhodamin 123 transport (16).

\section{Recombinant cytosolic domains of Pgp: design, overexpression in bacteria and purification}

The main difficulty in establishing Pgp structure-function relationships is the low

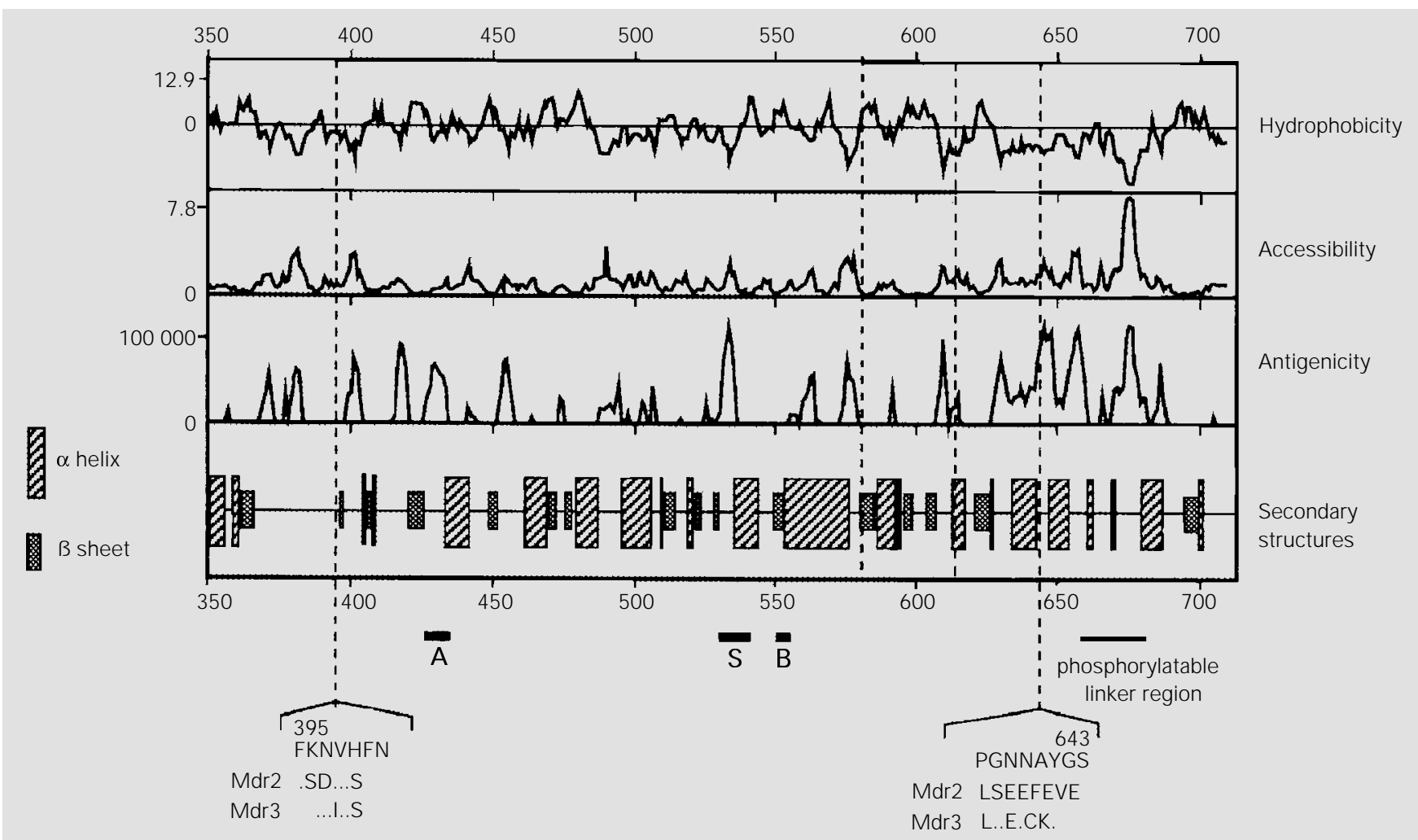

Figure 2 - Determination of molecular boundaries of recombinant NBD from the prediction of hydrophobicity, accessibility, antigenicity and secondary structures. The 350-708-amino acid sequence from mouse P-glycoprotein encoded by the mdrl gene was analyzed using the ANTHEPROT program (54). Mdr, Multidrug resistance. 
amount of pure protein available for studies. In natural sources, the protein is present at low concentrations and no efficient way to heterologously overexpress Pgp has yet been found. An additional problem is protein purification which is, in general, tedious and time-consuming for membrane proteins. A suitable alternative way to address this problem was to attempt the overexpression of the recombinant cytosolic NBDs which have been predicted to be soluble. We have decided to work with mdr1 Pgp from mouse adrenal glands where the corresponding mRNAs are expressed at above-average levels.

Four different recombinant domains were prepared: i) $N$-terminal NBD1, ii) extended NBD1 including a phosphorylatable linker sequence, iii) $C$-terminal NBD2, and iv) shorter NBD2 (cf. Figures 3 and 4). cDNAs corresponding to the different domains were obtained by reverse-transcriptase PCR from polyA ${ }^{+}$mRNAs purified from total RNAs of mouse adrenal glands using specific oligonucleotide primers. Alternatively, fragments of the complete mdr1 cDNA (provided by Pr. P. Gros, Montreal, Canada (5)) were amplified by direct PCR and cloned into inducible bacterial expression plasmids. Protein expression was achieved using Escherichia coli strains JM105, JM109 or BL21 (DE3).

The domain limits were predicted using the ANTHEPROT software (54), as illustrated in Figure 2 for NBD1. The domains were designed taking into account the following aspects: 1) all domains contained the Walker motifs $\mathrm{A}$ and $\mathrm{B}$, and the $\mathrm{S}$ signature since they are characteristic for ABC transporters, 2) the hydrophobicity was predicted and minimized to increase protein solubility and to aid protein purification, 3 ) secondary structure elements were predicted and sequence boundaries were chosen to be outside the helical or strand regions to avoid structural disturbance of the recombinant protein, 4) designed oligonucleotides had to be sufficiently different from the corresponding regions of the $m d r 2$ and $m d r 3$ genes, also present in adrenal glands, to avoid a crossreaction.

Three different lengths of the NBD1 were designed in this way, with the $N$-terminal end set at Asn-395 and the $C$-terminal one at either Ser-643, Glu-613 or Thr-581. Extended NBD1 stretched from Glu-371 to Glu-705, including the phosphorylatable linker region and Trp-696. A long NBD2 was designed from Lys-1025 to $C$-terminal Ser-1276, and a shorter one from Thr-1044 to Thr-1224, both proteins containing Trp-1106 (Figure $3, \mathrm{c}$ and d). A schematic sequence alignment of the different recombinant domains with HisP, a cytosolic subunit of an ABC transporter whose 3-D structure has recently been reported (10), is shown in Figure 4.

\section{Binding of nucleotides and analogues}

The NBDs 1 were obtained as fusion proteins with an $N$-terminal hexahistidine tag. The cDNAs were cloned into pQE-30 and protein expression was induced by isopropyl-1-thio-ß-D-galactopyranoside (IPTG). Protein purification involved cell lysis by

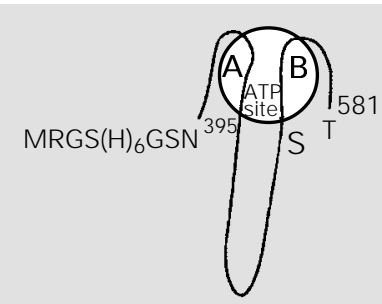

(a) NBD1

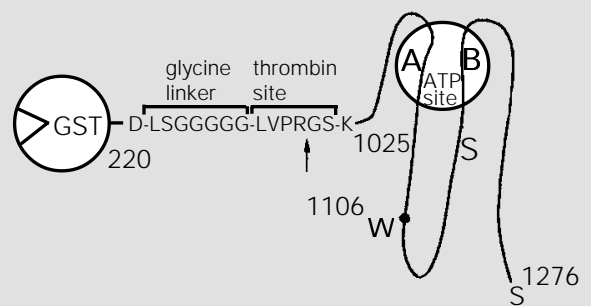

(c) GST-NBD2

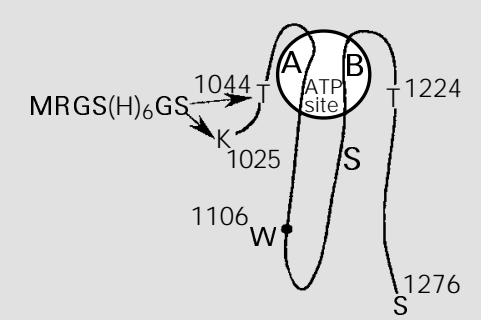

(d) NBD2

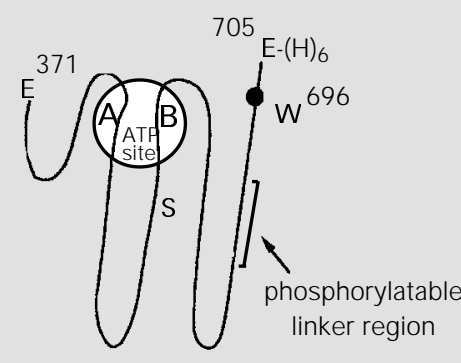

(b) extended NBD1
Figure 3 - Recombinant cytosolic domains of P-glycoprotein. 
French press treatment and affinity chromatography on a nickel-nitrilotriacetic acid column (55). The amount of soluble protein present in the supernatant indicated that, in the case of the three different NBD1s, the protein yield was indirectly correlated to the length of the protein domain. Therefore, the shorter domain, from Asn-395 to Thr-581, was prepared in high amounts for biochemical and biophysical studies.

The domain exhibited a low, but significant, ATPase activity with an extrapolated maximal rate of $25 \mathrm{nmol} / \mathrm{min} \times \mathrm{mg}$ protein and a $\mathrm{K}_{\mathrm{m}}(\mathrm{ATP})$ of $2.1 \mathrm{mM}$. A similar rate was obtained for the recombinant nucleotide-binding fold of CFTR. The activity of Pgp NBD1 was competitively inhibited by micromolar concentrations of the 2',3'-O(2,4,6-trinitrophenyl) (TNP) derivative of ATP, a high-affinity fluorescent nucleotide analogue $\left(\mathrm{K}_{\mathrm{D}}=2.2 \mu \mathrm{M}\right.$ by enhancement of extrinsic fluorescence) whose structure is shown in Figure 5a. In addition, chemical modification by $N$-ethylmaleimide of the single cysteine residue, present in the Walker motif $A$, increased the $\mathrm{K}_{\mathrm{m}}$ (ATP) by 6-fold. This is probably due to steric hindrance caused by the modification, since the corresponding serine residue in HisP forms a hydrogen bond with the B-phosphate of ATP (10). The NBD1 was labelled upon photoirradiation in the presence of radioactive 8.7 $\mu \mathrm{M} 8$-azido-ATP and $5 \mathrm{mM} \mathrm{MgCl}_{2}$, possibly by derivatizing Tyr-400 according to results obtained with the full-length Pgp (56). Since NBD1 did not contain any tryptophan residue, the quenching of intrinsic fluorescence due to tyrosine residues was monitored to study the binding of 2'(3')-N-methylanthraniloyl (MANT) derivative of ATP (Figure $5 b$ ), a fluorescent high-affinity nucleotide analogue $\left(K_{D}=25 \mu \mathrm{M}\right)$. There was evidence that the maximal quenching of about 15$20 \%$ was due at least in part to an interaction with Tyr-464. When the NBD1 and NBD2 sequences of the same protein are aligned, Tyr-464 corresponds to Trp-1106 which was shown to interact with the MANT group of MANT-ATP (see below). The competition observed between ATP and its analogues, TNP-ATP and MANT-ATP, indicates that all three nucleotides use the same binding site.

\section{Interaction with hydrophobic steroids}

Extended NBD1 was fused to a $C$-terminal hexahistidine tag and was overexpressed after insertion of its DNA into a pT7-7 plasmid. Despite many attempts to increase protein solubility, such as lowering the temperature for bacterial growth and varying the IPTG concentration, recombinant extendedNBD1 was exclusively found in inclusion bodies. A soluble active form of the ex-
Figure 4 - Schematic sequence alignment of the recombinant cytosolic domains of mouse mdr1 P-glycoprotein with HisP, a subunit of bacterial histidine permease, of which the 3-D structure has been determined (10). In this alignment, the position of the Walker A motif was fixed, and secondary structure elements of HisP are shown in the upper part of the Figure. The side chain of $\mathrm{Y}^{16}$ of HisP is involved in a stacking interaction with the adenine ring of ATP.

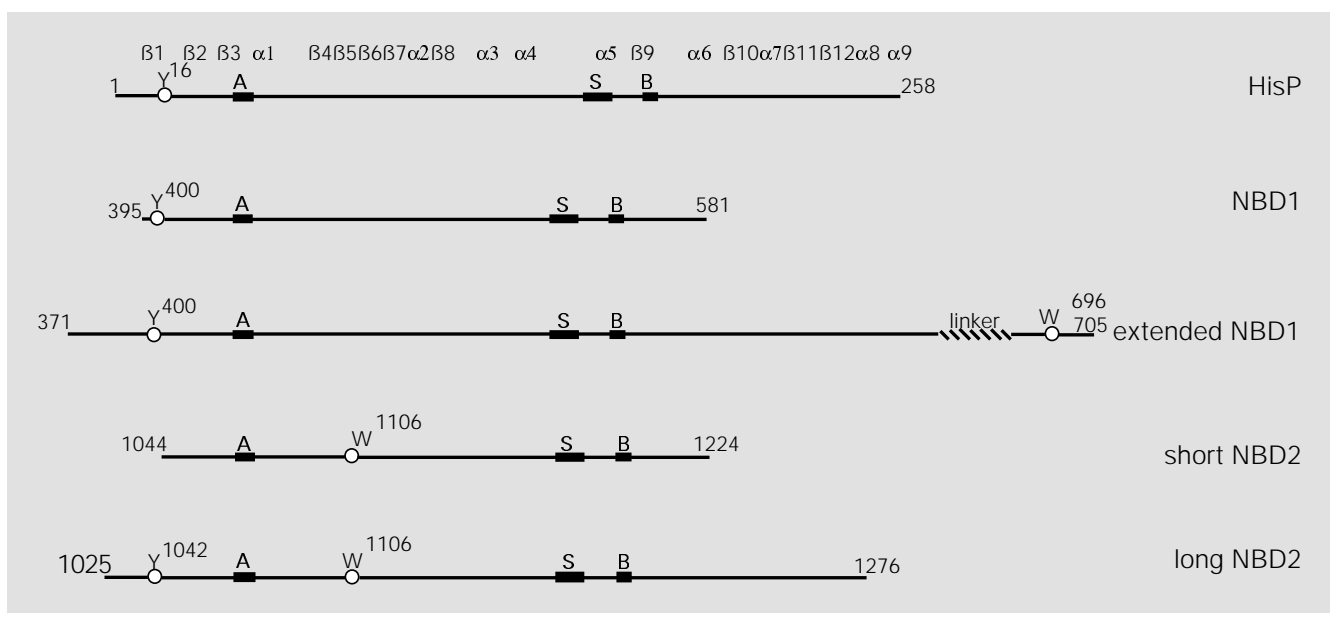


tended NBD1 was obtained when the protein was solubilized from inclusion bodies. This procedure involved solubilization with a rather low $(1.5 \mathrm{M})$ guanidine concentration and a quick 20 -fold dilution in phosphate buffer, $\mathrm{pH} 9$, containing $0.5 \mathrm{M} \mathrm{NaCl}, 10-20 \%$ glycerol, and $0.01 \%$ 6-O-(N-heptylcarbamoyl)-methyl- $\alpha$-D-glucopyranoside (HECAMEG). There are two pieces of evidence for the guanidine-solubilized extended NBD1 being correctly folded: 1) NBD1 and extended NBD1 had similar binding affinities for nucleotides and analogues, and 2) NBD1 purified from inclusion bodies and the same protein purified from the supernatant had similar properties (57). Trp-696 exhibited fluorescence properties characteristic for being in a quite hydrophobic environment, with maximal emission at a relatively low wavelength, $325 \mathrm{~nm}$ upon excitation at $295 \mathrm{~nm}$; this further confirmed accurate folding, at least around Trp-696. Interaction with MANT-ATP produced both a quenching of extended NBD1 intrinsic fluo- rescence and a fluorescence resonance energy transfer between Trp-696 and the MANT group of bound MANT-ATP. The $57 \%$ maximal efficiency indicated that donor and acceptor fluorophores were at a distance of about 17-23 $\AA$ (57).

The quenching of Trp-696 fluorescence was also used to monitor interaction with steroids. A marked effect was observed with hydrophobic steroids, known not to be transported by Pgp, with the following efficiency: RU 486 (cf. Figure 5c), megestrol acetate > $\Delta^{6}$-progesterone $>$ medroxyprogesterone acetate $>$ progesterone $>5 \beta$-pregnanedione. In contrast, a very low interaction was detected with hydrophilic hydroxylated derivatives, known to be transported by Pgp: triamcinolone $<$ cortisol, dihydrotestosterone, corticosterone, $11 \alpha$-hydroxyprogesterone $<$ testosterone, dexamethasone, $17 \alpha$-hydroxyprogesterone, triamcinolone acetonide $<11$ deoxycorticosterone. The same preference was observed for the binding of these compounds to full-length Pgp. The overall re-

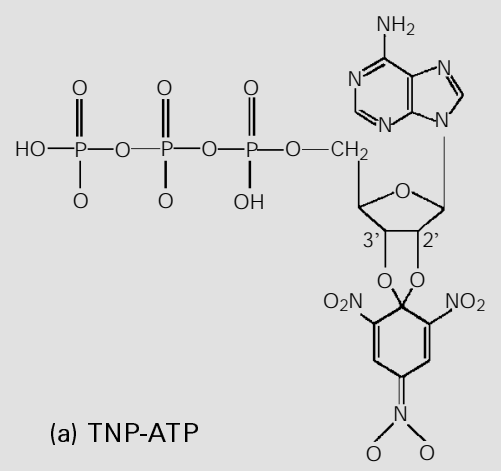

(a) TNP-ATP

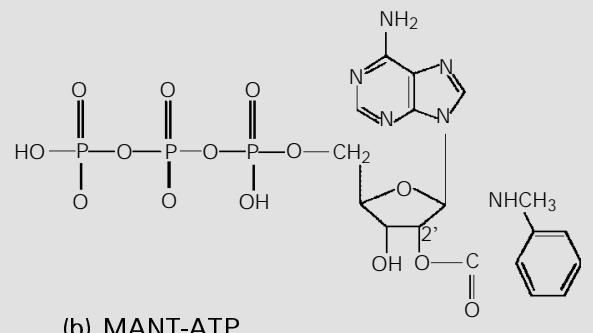

(b) MANT-ATP

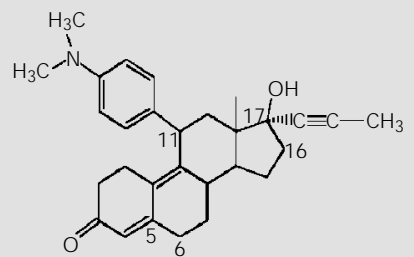

(c) RU 486

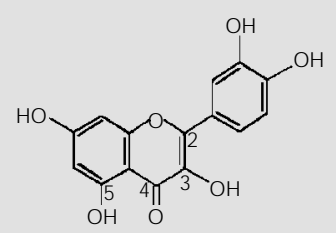

(d) Quercetin
Figure 5 - Chemical structure of compounds which bind to cytosolic NBDs. TNP, 2' $3^{\prime}-\mathrm{O}-(2,4$, 6-trinitrophenyl); MANT, 2'(3')$\mathrm{N}$-methylanthraniloyl. 


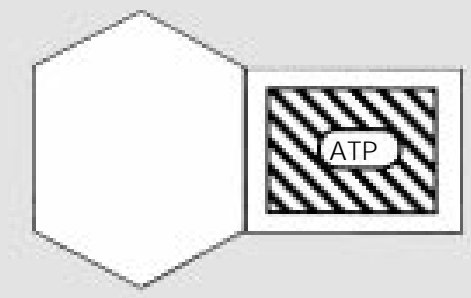

A: ATP binding

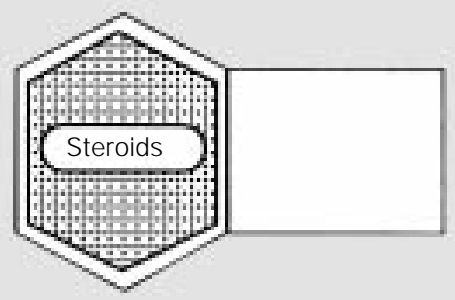

B: Interaction with drugs or modulators

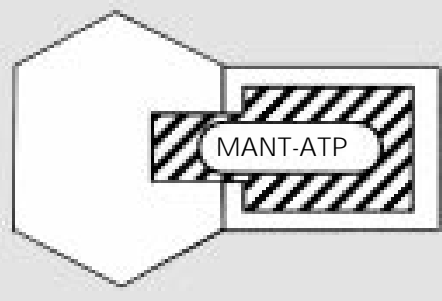

C: MANT-ATP binding

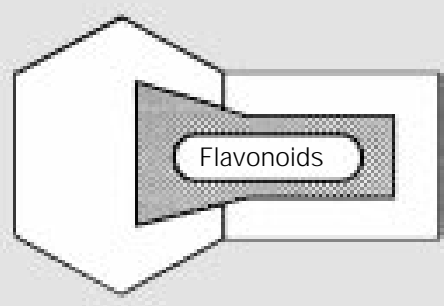

D: Overlapping of both sites by flavonoids
Figure 6 - Proposed schematic model of NBDs showing the relative positions of different nucleotide- and effector-binding sides. MANT, 2' (3')-N-methylanthraniloyl.

sults indicate that hydrophobic substituents increase steroid binding affinity for Pgp, prevent their transport and increase their ability to inhibit drug efflux, whereas hydroxyl groups at positions 11,16, 17 and 21 reduce binding affinity and inhibitory effects and allow the steroids to be transported (45).

The almost complete quenching of extended NBD1 intrinsic fluorescence by RU 486 suggested that Trp-696 is located within, or in close proximity to, the steroid-interacting region. The quenching was unaffected by preincubation with $10 \mathrm{mM} \mathrm{ATP}$, indicating that the two sites are distinct. In contrast, a complete antagonism was observed with MANT-ATP since its binding, as monitored by enhancement of extrinsic fluorescence, was prevented by preincubation with RU 486, and bound MANT-ATP was displaced upon addition of the antiprogestin. This supports the existence of a cytosolic steroidinteracting region adjacent to the ATP-binding site (Figure 6, A and B). The mutually exclusive binding of MANT-ATP against
RU 486 could be due to overlapping of the steroid-interacting region by the hydrophobic MANT group of the nucleotide analogue bound to the ATP site (Figure 6C), although a distant conformational change cannot be completely excluded.

\section{Bifunctional interactions with flavonoids}

The cDNA of NBD2 was cloned into pGEX-KT, and the domain was obtained as a glutathione $S$-transferase fusion protein (cf. Figure $3 \mathrm{~b}$ ) which was purified on a glutathione-Sepharose 4B column. The protein was found to bind ATP and its highaffinity derivative, TNP-ATP (58). However, the NBD2 appeared to be quite unstable when separated from the glutathione $S$-transferase after thrombin cleavage, and thus could not be extensively studied. NBD2 was then generated as an $N$-terminally tagged hexahistidine fusion protein and was found to be soluble and stable under the conditions mentioned above, which allowed the preparation of large amounts of pure protein. The solubility appeared to be higher for a shorter version (from Thr-1044 to Thr-1224) of the domain (cf. Figure 3d). The intrinsic fluorescence of the domain due to the single Trp1106 was highly quenched with MANTATP, showing also an efficient fluorescence resonance energy transfer. A similar NBD2 from Chinese hamster ovary Pgp was obtained in fusion to maltose-binding protein.

NBD2 intrinsic fluorescence was also highly quenched upon interaction with flavonoids (59). The binding affinities of different classes of flavonoids could be ranked in the following order: flavones (quercetin (cf. Figure 5d), apigenin) $>$ flavanones (naringenin) $>$ isoflavone $($ genistein $)>$ glycosyl-flavones (rutin). Within flavones, hydroxyl groups at positions 3 and 5 in close proximity to the ketone at position 4 markedly increased the binding affinity. They might contribute to a more precise mimick- 
ing of the adenine moiety of ATP, which has already been observed for the flavonoidliganded 3-D structures of cycline-dependent kinase 2 (52) and Hck tyrosine kinase (53). Flavonoid binding to NBD2 was indeed partly prevented by preincubation with ATP or displaced upon ATP addition (59). The binding affinity was further increased by disrupting the $\mathrm{C}$ ring to give chalcones, and by substituting the $\mathrm{B}$ ring with a hydrophobic halogen atom (60). In addition, flavonoid binding also partly antagonized the binding of the steroid RU 486. Since the steroid-interacting region is assumed to be close to the ATP site, bound flavonoids most likely cover both the ATP site and the vicinal steroid site. Therefore, flavonoids constitute a new class of MDR modulators which undergo bifunctional interactions with the cytosolic domains of Pgp (Figure 6D).

\section{Interest of recombinant cytosolic domains for the study of Pgp struc- ture-function relationships and for drug design}

In addition to the ATP-binding site, NBDs also contain sequence segments which are likely to carry out other physiological func- tions, such as signal transduction. The study of proteins mutated around the Walker motif B in Pgp, or Ste6 or malK, provided evidence that drug binding and ATP hydrolysis generate cellular signals. NBDs also contain part of drug-binding sites as demonstrated by photoaffinity labelling with either iodoaryl azidoprazosin or 3'- $p$-benzoyldihydrocinnamoyl-taxol. The similar behavior of the two NBDs towards steroids and especially towards flavonoids supports the existence of 2 modulator-binding sites (Figure 6). This is consistent with conclusions drawn from numerous kinetic and labelling experiments of membrane-inserted Pgp with modulators and drug substrates.

The overall results indicate that Pgp NBDs are active and can be prepared in high amounts as purified proteins which permit crystallization trials. The presence of specific binding sites for ATP offers the possibility to search for selective bifunctional modulators able to cover both the ATP-binding site and the vicinal steroid-interacting region. Identification of reactive residues at both binding sites might allow a more rational design and synthesis of highly potent and selective inhibitors.

\section{References}

1. Gottesman MM \& Pastan I (1993). Biochemistry of multidrug resistance mediated by the multidrug transporter. Annual Review of Biochemistry, 62: 385-427.

2. EndicottJ A \& Ling V (1989). The biochemistry of P-glycoprotein-mediated multidrug-resistance. Annual Review of Biochemistry, 58: 137-171.

3. Ruetz S \& Gros P (1994). Phosphatidylcholine translocase: a physiological role for the mdr2 gene. Cell, 77: 1071-1081.

4. van Helvoort $A$, Smith $A J$, Sprong $H$, Fritzche I, Schinkel AH, Borst $P$ \& van Meer G (1996). MDR1 P-glycoprotein is a lipid translocase of broad specificity, while MDR3 P-glycoprotein specifically translocates phosphatidylcholine. Cell, 87: 507517.
5. Gros P, Croop J \& Housman D (1986). Mammalian multidrug resistance gene: complete sequence indicates strong homology to bacterial transport proteins. Cell, 47: 371-380.

6. Kast C, Canfield V, Levenson R \& Gros P (1996). Transmembrane organization of mouse P-glycoprotein determined by epitope insertion and immunofluorescence. J ournal of Biological Chemistry, 271: 9240-9248.

7. Walker JE, Saraste M, Runswick MJ \& Gay NJ (1982). Distantly related sequences in the $\alpha$-and ß-subunits of ATP synthase, myosin, kinases and other ATPrequiring enzymes and a common nucleotide binding fold. EMBO J ournal, 1: 945951.
8. Higgins CF, Hiles ID, Salmond GPC, Gill DR, Downie J A, Evans IJ , Holland IB, Gray L, Buckel SD, Bell W \& Hermodson MA (1986). A family of related ATP-binding subunits coupled to many distinct biological processes in bacteria. Nature, 323: 448-450.

9. Rosenberg MF, Callaghan R, Ford RC \& Higgins CF (1997). Structure of the multidrug resistance P-glycoprotein to $2.5 \mathrm{~nm}$ resolution determined by electron microscopy and image analysis. J ournal of Biological Chemistry, 272: 10685-10694.

10. Hung L-W, Wang IX, Nikaido K, Liu P-Q, Ames GF-L \& Kim S-H (1998). Crystal structure of the ATP-binding subunit of an ABC transporter. Nature, 396: 703-707.

11. Loo TW \& Clarke DM (1994). Reconstitu- 
tion of drug-stimulated ATPase activity following co-expression of each half of human P-glycoprotein as separate polypeptides. J ournal of Biological Chemistry, 269: 7750-7755.

12. Azzaria M, Schurr E \& Gros P (1989). Discrete mutations introduced in the predicted nucleotide-binding sites of the mdrl gene abolish its ability to confer multidrug resistance. Molecular and Cellular Biology, 9: 5289-5297.

13. Senior AE \& Bhagat S (1998). P-glycoprotein shows strong catalytic cooperativity between the two nucleotide sites. Biochemistry, 37: 831-836.

14. Leveille-Webster CR \& Arias IM (1995). The biology of the P-glycoproteins. J ournal of Membrane Biology, 143: 89-102.

15. Tang-Wai DF, Brossi A, Amold LD \& Gros $P$ (1993). The nitrogen of the acetamido group of colchicine modulates P-glycoprotein-mediated multidrug resistance. Biochemistry, 32: 6470-6476.

16. Shapiro AB \& Ling V (1997). Positively cooperative sites for drug transport by P-glycoprotein with distinct drug specificities. European J ournal of Biochemistry, 250: 130-137.

17. Bolhuis $H$, van Veen HW, Poolman B, Driessen AJ M \& Konings WN (1997). Mechanisms of multidrug transporters. FEMS Microbiology Reviews, 21: 55-84.

18. Higgins CF \& Gottesman MM (1992). Is the multidrug transporter a flippase? Trends in Biochemical Sciences, 17: 1821.

19. Stein WD (1997). Kinetics of the multidrug transporter (P-glycoprotein) and its reversal. Physiological Reviews, 77: 545590.

20. Schinkel $A H$, Smit JJ $M$, van Tellingen $O$, Beijnen J H, Wagenaar $E$, van Deemter $L$, Mol CAAM, van der Valk MA, RobanusMaandag EC, Riele HPJ, Bern AJM \& Borst P (1994). Disruption of the mouse mdrla P-glycoprotein gene leads to a deficiency in the blood-barrier and to increased sensitivity to drugs. Cell, 77: 491502.

21. Ueda K, Okamura N, Hirai M, Tanigawara Y, Saeki T, Kioka N, Komano T \& Hori R (1992). Human P-glycoprotein transports cortisol, aldosterone, and dexamethasone, but not progesterone. J ournal of Biological Chemistry, 267: 24248-24252.

22. Wolf DC \& Horwitz SB (1992). P-glycoprotein transports corticosterone and is photoaffinity-labelled by the steroid. International J ournal of Cancer, 52: 141146.

23. Bruggemann EP, Germann UA,
Gottesman MM \& Pastan I (1989). Two different regions of P-glycoprotein are photoaffinity labeled by azidopine. J ournal of Biological Chemistry, 264: 1548315488.

24. Evans GL, Ni B, Hrycyna CA, Chen D, Ambudkar SV, Pastan I, Germann UA \& Gottesman MM (1995). Heterologous expression systems for P-glycoprotein: E. coli, yeast and baculovirus. Journal of Bioenergetics and Biomembranes, 27: 4352.

25. Germann UA, Chambers TC, Ambudkar SV, Pastan I \& Gottesman MM (1995). Effects of phosphorylation of P-glycoprotein on multidrug resistance. J ournal of Bioenergetics and Biomembranes, 27: 5360.

26. Smith CD \& Zilfou J T (1995). Circumvention of P-glycoprotein-mediated multiple drug resistance by phosphorylation modulators is independent of protein kinases. J ournal of Biological Chemistry, 270: 28145-28152.

27. Germann UA, Chambers TC, Ambudkar SV, Licht T, Cardarelli CO, Pastan I \& Gottesman MM (1996). Characterisation of phosphorylation-defective mutants of human P-glycoprotein expressed in mammalian cells. J ournal of Biological Chemistry, 271: 1708-1716.

28. Doige CA \& Ames GF-L (1993). ATP-dependent transport systems in bacteria and humans: relevance to cystic fibrosis and multidrug resistance. Annual Review of Microbiology, 47: 291-319.

29. Higgins CF (1992). ABC transporters: From microorganisms to man. Annual Review of Cellular Biology, 8: 67-113.

30. Deeley RG \& Cole SPC (1997). Function, evolution and structure of multidrug resistance protein (MRP). Seminars in Cancer Biology, 8: 193-204.

31. Welsh MJ, Robertson AD \& Ostedgaard LS (1998). The $A B C$ of a versatile engine. Nature, 396: 623-624.

32. Borst $P$, Kool $M \&$ \& Evers $R$ (1997). Do CMOAT (MRP2), other MRP homologues, and LRP play a role in MDR? Seminars in Cancer Biology, 8: 205-213.

33. Balzi E \& Goffeau A (1991). Multiple or pleiotropic drug resistance in yeast. Biochimica et Biophysica Acta, 1073: 241252.

34. Decottignies A \& Goffeau A (1997). Complete inventory of the yeast $A B C$ proteins. Nature Genetics, 15: 137-145.

35. Rubio J P \& Cowman AF (1996). The ATP binding cassette $(A B C)$ gene family of Plasmodium falciparum. Parasitology Today, 12: 135-140.
36. Foote SJ , Thompson J K, Cowman AF \& Kemp DJ (1989). Amplification of the multidrug resistance gene in some chloroquine-resistant isolates of $P$. falciparum. Cell, 57: 921-930.

37. Ouelette $M$, Fase-Fowler $F \&$ Borst $P$ (1990). The amplified $\mathrm{H}$ circle of methotrexate-resistant Leishmania tarentolae contains a novel P-glycoprotein gene. EMBO J ournal, 9: 1027-1033.

38. Dallagiovanna B, Gamarro F \& Castanys $S$ (1996). Molecular characterization of a P-glycoprotein-related tcpgp2 gene in Trypanosoma cruzi. Molecular and Biochemical Parasitology, 75: 145-157.

39. Ullman B (1995). Multidrug resistance and P-glycoproteins in parasitic protozoa. J ournal of Bioenergetics and Biomembranes, 27: 77-84.

40. Chiquero MJ, Perez-Victoria J M, O'Valle F, Gonzalez-Ros J M, del Moral RG, Ferragut J A, Castanys $S \&$ Gamarro $F$ (1998). Altered drug membrane permeability in a multidrug-resistant Leishmania tropica line. Biochemical Pharmacology, 55: 131-139.

41. van Veen HW, Callaghan R, Soceneantu L, Sardini A, Konings WN \& Higgins CF (1998). A bacterial antibiotic-resistance gene that complements the human multidrug-resistance P-glycoprotein gene. Nature, 391: 291-295.

42. Sikic BI (1997). Pharmacologic approaches to reversing multidrug resistance. Seminars in Hematology, 34: 40-47.

43. ZamoraJ M, Pearce HL \& Beck WT (1988) Physiological-chemical properties shared by compounds that modulate multidrug resistance in human leukemic cells. Molecular Pharmacology, 33: 454-562.

44. Seelig A (1998). A general pattern for substrate recognition by P-glycoprotein. European J ournal of Biochemistry, 251: 252261.

45. Barnes KM, Dickstein B, Culter GB, Fojo T $\&$ Bates SE (1996). Steroid transport, accumulation, and antagonism of P-glycoprotein in multidrug-resistant cells. Biochemistry, 35: 4820-4827.

46. Gruol DJ , Zee MC, Trotter J \& Bourgeois $S$ (1994). Reversal of multidrug resistance by RU 486. Cancer Research, 54: 30883091.

47. Callaghan R \& Higgins CF (1995). Interaction of tamoxifen with the multidrug resistance P-glycoprotein. British J ournal of Cancer, 71: 294-299.

48. Middleton E \& Kandaswami C (1993). The impact of plant flavonoids on mammalian biology: implications for immunity, inflammation and cancer. In: Harborne J B (Edi- 
tor), The Flavonoids: Advances in Research Since 1986. Chapman \& Hall, London, 619-652.

49. Critchfield J W, Welsh CJ, Phang J M \& Yeh GC (1994). Modulation of adriamycin in accumulation and efflux by flavonoids in HCT-15 colon cells. Activation of P-glycoprotein as a putative mechanism. Biochemical Pharmacology, 48: 14371445.

50. Scambia G, Ranelletti FO, Benedetti Panici P, De Vincenzo R, Bonanno G, Ferrandina G, Piantelli M, Bussa S, Rumi C, Cianfriglia M \& Mancuso S (1994). Quercetin potentiates the effect of adriamycin in a multidrug-resistant M CF-7 human breast-cancer cell line: P-glycoprotein as a possible target. Cancer Chemotherapy and Pharmacology, 34: 459-464.

51. Shapiro AB \& Ling V (1997). Effect of quercetin on Hoechst 33342 transport by purified and reconstituted P-glycoprotein. Biochemical Pharmacology, 53: 587-596.

52. De Azevedo WF, Mueller-Dieckmann $\mathrm{H}-\mathrm{J}$, Schulze-Gahmen U, Worland PJ , Sausville E \& Kim S-H (1996). Structural basis for specificity and potency of a flavonoid inhibitor of human CDK2, a cell cycle kinase. Proceedings of the National Academy of Sciences, USA, 93: 2735-2740.

53. Sicheri F, Moarefi I \& Kuryan J (1997). Crystal structure of the Src family tyrosine kinase Hck. Nature, 385: 602-609.

54. Geourjon C \& Deléage G (1995). ANTHEPROT 2.0: a three-dimensional module fully coupled with protein sequence analysis methods. J ournal of Molecular Graphics, 13: 209-212.

55. Dayan G, Baubichon-Cortay H, J ault J -M, Cortay J -C, Deléage G \& Di Pietro A (1996). Recombinant N-terminal nucleotide-binding domain from mouse P-glycoprotein: overexpression, purification and role of cysteine-430. J ournal of Biological Chemistry, 271: 11652-11658.

56. Sankaran B, Bhagat S \& Senior AE (1997). Photoaffinity labelling of P-glycoprotein catalytic sites. FEBS Letters, 417: 119122.

57. Dayan G, J ault J -M, Baubichon-Cortay $H$, Baggetto LG, RenoirJ M, Baulieu EE, Gros P \& Di Pietro A (1997). Binding of steroid modulators to recombinant cytosolic domain from mouse P-glycoprotein in close proximity to the ATP site. Biochemistry, 36: 15208-15215.

58. Baubichon-Cortay H, Baggetto LG, Dayan $G \&$ Di Pietro A (1994). Overexpression and purification of the carboxyl-terminal nucleotide-binding domain from mouse P-glycoprotein. Strategic location of a tryptophan residue. J ournal of Biological Chemistry, 269: 22983-22989.

59. Conseil G, Baubichon-Cortay H, Dayan G, J ault J -M, Barron D \& Di Pietro A (1998). Flavonoids: a class of modulators with bifunctional interactions at vicinal ATPand steroid-binding sites on mouse P-glycoprotein. Proceedings of the National Academy of Sciences, USA, 95: 9831-9836.

60. Bois F, Beney C, Boumendjel A, Mariotte A-M , Conseil G \& Di Pietro A (1998). Halogenated chalcones with high-affinity binding to P-glycoprotein: potential modulators of multidrug resistance. J ournal of Medicinal Chemistry, 41: 4161-4164. 